\title{
1 Novel feature selection methods for construction of accurate
}

\section{2 epigenetic clocks}

Sinclair

\section{Affiliations}

Blavatnik Institute, Dept. of Genetics, Paul F. Glenn Center for Biology of Aging Research at

Harvard Medical School, Boston, MA

$\underline{\text { Abstract: }}$

Epigenetic clocks allow the accurate prediction of age based on the methylation status of specific

CpG sites in a variety of tissues. These predictive models can be used to distinguish the biological age of an organism from its chronological age, and are a powerful tool to measure the effectiveness of aging interventions. There is a growing need for methods to efficiently construct epigenetic clocks. The most common approach is to create clocks using elastic net regression modelling of all measured CpG sites, without first identifying specific features or CpGs of interest. The addition of feature selection approaches provides the opportunity to reduce the cost and time of clock development by decreasing the number of $\mathrm{CpG}$ sites included in clocks. Here, we apply both classic feature selection methods and novel combinatorial methods to the development of epigenetic clocks. We perform feature selection on the human whole blood methylation dataset of $\sim 470,000 \mathrm{CpG}$ features published by Hannum and colleagues (2015). We develop clocks to predict age, using a variety of feature selection approaches, and all clocks have R2 correlation scores of greater than 0.73 . The most predictive clock uses $35 \mathrm{CpG}$ sites for a R2 correlation score of 0.87 . The five most frequent sites across all clocks are also modelled to build a clock with a R2 correlation score of 0.83 . These two clocks are validated on two external datasets where they 
maintain excellent predictive accuracy and outperform Hannum et al's model in accuracy of age

prediction despite using significantly less CpGs. We also identify the associated gene regulatory regions

of these $\mathrm{CpG}$ sites, which may be possible targets for future aging studies. These novel feature selection

algorithms will lower the number of sites needed to be sequenced to build clocks and allow

conventionally expensive aging epigenetic studies to cost a fraction of what it would normally.

\section{Introduction:}

Epigenetic clocks allow for the prediction and observation of biological aging (Bocklandt, 2011). slow or reverse aging the demand for more robust, accurate clocks is growing.

The first epigenetic clocks were created by Bocklandt et al (Bocklandt et al, 2011) and quickly followed by the Hannum and Horvath labs in 2013 (Hannum et al, 2013; Horvath 2013). The Hannum clock, based on methylation analysis of DNA from peripheral blood mononuclear cells, was developed using elastic net regression modelling. 71 markers were selected from over 470,000 CpG sites to derive an age prediction accuracy of four years (Hanuum et al. 2013). Horvath's clock encompasses multiple tissue types and includes $353 \mathrm{CpG}$ sites that strongly predict age (Horvath 2013). Recently, there has been a focus on creating clocks with fewer $\mathrm{CpG}$ sites to enable epigenetic age profiling without the use of costly microarrays or expensive reduced-representation bisulfite sequencing (Ito el al. 2018, Park JL, et al. 2016, Zbieć-Piekarska et al. 2014, Spólnicka, M. et al. 2017). Alghanim et al.'s clock, built on blood 
methylation data, only uses $\mathrm{CpG}$ sites from three gene regions to explain $84-85 \%$ of age variance (Alghanim et al. 2017), and Weidner's clock based on only $3 \mathrm{CpG}$ sites, is able to predict age with an error of less than five years (Weidner et al., 2014).

Few epigenetic clock studies employ a discrete step to find optimal features for building clocks.

Feature selection is commonly used in situations where the number of features far outnumber the number

of samples (Guyon et al. 2003). Given the vast number of CpG sites in the genome and the relatively low number of samples in most studies, feature selection methods will improve the efficiency of clock building. Currently, the most common approach for clock building is to use a 'correlation-with-age' method, where CpGs that have a non-zero coefficient in ElasticNet Regression analyses are given more predictive power in the model (Horvath 2013, Hannum et al. 2013). Some clocks utilize more advanced feature selection methods such as Boruta (Renner et al., 2013), recursive feature selection (Wang et al., 2018; Darst, Malecki and Engelman, 2018; Meng, Murrelle and Li, 2008) or neural networks (Spólnicka et al. 2017). These algorithms select even fewer $\mathrm{CpG}$ features whilst still accurately predicting age.

However the number of clocks being built with these tools is minimal and there is more room to optimise

67 feature selection methods and parameters.

low numbers of CpG sites on the publicly available Hannum dataset (GSE40279), and evaluate their accuracy and generalizability on other datasets: GSE52588 (Horvath et al, 2015), GSE137688 (McEwen 2019), GSE85311 (Martens et al, 2020). We use a combination of modified standard methods that are readily available in python packages as well as the development of our own novel selection methods. We combine methods and use them in tandem to form new methods of feature selection, and optimise the development of epigenetic clocks to predict age.

\section{$\underline{\text { Results: }}$}


In order to test how few $\mathrm{CpG}$ sites could be selected while retaining predictive accuracy, we applied each of our feature selection methods to the Hannum methylation dataset (GSE40279). Table 1 and Figure 1 summarise the results of the feature selection approaches, including the number of $\mathrm{CpG}$ sites identified with each approach, and the correlation (r2) with chronological age on a test set. The best model for age prediction for this dataset is SelectKBest for 2000 features followed by Boruta. This approach selects $35 \mathrm{CpG}$ sites, with an $\mathrm{r} 2$ of 0.873 and a median absolute error of 3.08 years (Table 1).

\begin{tabular}{|c|c|c|c|c|}
\hline & $\begin{array}{l}\text { Average R2 } \\
\text { Score (from } \\
10 \mathrm{CV} \text { ) }\end{array}$ & STD (Years) & $\begin{array}{l}\text { Mean Absolute } \\
\text { Error (Years) }\end{array}$ & $\begin{array}{l}\text { Median } \\
\text { Absolute Error } \\
\text { (Years) }\end{array}$ \\
\hline KBest 2000 de novo then Boruta (35) & 0.873 & 0.05 & 3.82 & 3.08 \\
\hline $\begin{array}{l}\text { Intersection of all methods per CV fold then } \\
\text { Boruta (102) }\end{array}$ & 0.865 & 0.06 & 3.9 & 3 \\
\hline KBest 25 de novo (36) & 0.862 & 0.06 & 3.96 & 3.14 \\
\hline Boruta de novo (53) & 0.861 & 0.06 & 3.95 & 3.08 \\
\hline$\%$-RFE de novo to 1500 then Boruta (52) & 0.835 & 0.07 & 4.35 & 3.57 \\
\hline ElasticNet de novo/No Feature Selection (276) & 0.827 & 0.06 & 4.64 & 3.91 \\
\hline$\%$-RFE de novo to $100(161)$ & 0.825 & 0.07 & 4.69 & 3.83 \\
\hline Top 10 Most Frequent (10) & 0.825 & 0.08 & 4.59 & 3.7 \\
\hline Top 5 Most Frequent (5) & 0.82 & 0.08 & 4.6 & 3.79 \\
\hline $\begin{array}{l}\% \text {-RFE de novo to } 10000 \text { then Genetic } \\
\text { Algorithm (54) }\end{array}$ & 0.818 & 0.08 & 4.61 & 3.76 \\
\hline SFM ElasticNet de novo then Boruta (7) & 0.813 & 0.07 & 4.7 & 3.71 \\
\hline
\end{tabular}




\begin{tabular}{|c|c|c|c|c|}
\hline Genetic Algorithm de novo (85) & 0.812 & 0.08 & 4.72 & 3.68 \\
\hline SFM ElasticNet de novo (16) & 0.81 & 0.07 & 4.74 & 3.84 \\
\hline$\%$-RFE de novo to 1500 then SFM (16) & 0.81 & 0.07 & 4.74 & 3.84 \\
\hline SFM ExtraTrees de novo (5) & 0.77 & 0.08 & 5.36 & 4.27 \\
\hline SFM ExtraTrees de novo then Boruta (5) & 0.77 & 0.08 & 5.36 & 4.271 \\
\hline Neural Network feature selection (65) & 0.76 & 0.08 & 5.65 & 4.79 \\
\hline $\begin{array}{l}\text { Post Feature Selection Intersection of all } \\
\text { methods (1) }\end{array}$ & 0.73 & 0.09 & 5.75 & 4.38 \\
\hline Variance Threshold de novo (2) & 0.02 & 0.02 & 11.9 & 10.61 \\
\hline
\end{tabular}

Our other feature selection methods, including most of the SelectFromModel (SFM) methods, the

genetic algorithms and several combinations of methods, achieve an accuracy of between 0.77 to 0.82

(Table 1). Despite being fundamentally different in their approach, these methods accomplish similar

results and plateau in the same range of scores (Figure 1). Further optimization of each of these methods

is needed to warrant their usage over other more successful methods.

ElasticNet de novo (Table 1, Figure 1) represents a model without any feature selection methods

for comparison to the other models. This model uses all $\sim 450,000$ features to train a model without any

pre-selection or iterative algorithms. The resulting clock from this approach is based on $276 \mathrm{CpGs}$, which

is a much higher number of CpGs than clocks developed with the feature selection methods (Table 1), 
de novo score remarkably well with no prior method being applied ( 0.861 and 0.862 respectively). These are the best performing solo feature selection methods.

Using the five most frequently-selected CpGs among all the methods to build a clock gave a correlation score of 0.83 and median absolute error of 3.79 years (Table 1). Table 2 shows the corresponding GeneIDs for these CpGs. The most frequent CpG site is cg16867657 (ELOVL2) and training a clock on this single feature results in a correlation score of 0.73 (Table 1). Overall, these results demonstrate that using feature selection methods accurate epigenetic clocks can be constructed with only a few CpGs.

\begin{tabular}{|l|l|}
\hline Most Frequent 5 CpG Sites & Associated GeneID \\
\hline $\operatorname{cg} 16867657$ & ELOVL2 \\
\hline $\operatorname{cg} 10501210$ & C1orf132 \\
\hline $\operatorname{cg} 22454769$ & FHL2 \\
\hline $\operatorname{cg} 04875128$ & OTUD7A \\
\hline $\operatorname{cg} 19283806$ & CCDC102B \\
\hline
\end{tabular}

Table 2. Table showing the 5 CpG sites that are chosen as most frequent predictors for aging and their associated gene symbols

We also tested a neural network approach for feature selection. An ElasticNet Regression model trained on the top 65 features selected by the neural network, has a moderate $r 2$ value of 0.76 .

Interestingly, only four of the 65 identified neural network CpGs overlap with the CpGs selected by other methods described here. Given a neural network's unique ability to detect these CpG sites as predictors, this is a promising predictive tool to uncover more obscure CpGs that most conventional methods miss. 
We selected two models developed above for further validation of their accuracy in independent datasets. SelectKBest for 2000 features followed by Boruta \& the top 5 most frequent features are the best

122 performing feature selection method and the clock with the lowest number of CpGs sets, respectively.

123 We applied these two clock models to two published blood methylation datasets. GSE85311 is methylation profiling of blood taken from young and old human subjects of varying exercise level (Martens et al, 2020). GSE52588 is methylation profiling of blood taken from subjects with and without down syndrome (Horvath et al, 2015). Each of the clocks predicted age very well in these external data sets with R2 values greater than 0.93 (Table 3, Figure 2), performing better than Hannum's final published clock created from $71 \mathrm{CpG}$ sites (Hannum 2013), despite using far fewer features.

\begin{tabular}{|l|l|l|l|l|}
\hline Feature Selection & Data set & r2 Score & Mean Absolute & Median Absolute \\
\hline KBethods & & & Error (Years) & Error (Years) \\
\hline then Boruta (35) & GSE85311 & 0.931 & 4.66 & 4.18 \\
\hline Top 5 Most Frequent & GSE85311 & 0.964 & 3.35 & 2.68 \\
\hline$(5)$ & GSE137688 & 0.710 & 2.0 & 1.6 \\
\hline & & & 5.71 & 5.60 \\
\hline & GSE52588 & 0.932 & 4.56 & 3.98 \\
\hline
\end{tabular}

Table 3. Table showing the results of the two final models trained on the Hannum dataset (GSE40279, 
Horvath et al, 2015), Martens exercise blood dataset (GSE85311, Martens et al, 2020), and buccal

dataset (GSE137688, McEwen 2019). Number of CpG sites/features in parentheses.

To test whether clocks developed with our feature selection approaches can be applied to datasets other than those developed in blood, the two selected models above were also applied to a buccal cell dataset (GSE137688, (McEwen 2019)). Using the methods on this dataset, we achieved a top r2 score of 0.71 with the SelectKBest for 2000 features followed by Boruta method and $\mathrm{r} 2$ of 0.47 with the Top 5 Most Frequent method (Table 3). The scores were expectedly lower than the results of the other two validation sets because the clocks were trained on blood data, and applied to buccal swab data, which have inherent sampling and variance differences. While the $\mathrm{r} 2$ scores were not as high, the models did have very low mean and median absolute errors; the lowest of all results in this paper. Given the abundance and ease of access buccal samples provide, this is promising rudimentary groundwork for the application of feature selection methods on sample types beyond blood.

We next wanted to test whether the features selected with our methods, could be used to make accurate clocks in other datasets. We took the CpGs selected from the Hannum dataset using our top two models (SelectKBest for 2000 followed by Boruta \& the top 5 most frequent CpG features), and selected those same CpGs in the Horvath down syndrome dataset (GSE52588, Horvath et al, 2015). Using only those CpGs, we created a clock from that remaining dataset, using the same cross-validation scheme (see Methods) used for the original Hannum experiment above. Remarkably, clocks developed in this dataset based on 35 features (SelectKBest for 2000 features followed by Boruta) and 5 features (top 5 most frequent) achieved $\mathrm{r} 2$ scores of 0.928 and 0.911 respectively (Table 4). This shows these CpGs can be used across datasets to create accurate clocks and are possibly universal, non-dataset specific CpGs for predicting age. 
bioRxiv preprint doi: https:/doi org/10.1101/2022.02.21.481326; this version posted February 22, 2022. The copyright holder for this preprint (which was not certified by peer review) is the author/funder, who has granted bioRxiv a license to display the preprint in perpetuity. It is made available under aCC-BY 4.0 International license.

\begin{tabular}{|l|l|l|l|}
\hline $\begin{array}{l}\text { Feature Selection } \\
\text { Method CpGs used }\end{array}$ & $\begin{array}{l}\text { Average R2 Score (from } \\
\mathbf{1 0 C V})\end{array}$ & $\begin{array}{l}\text { Mean Absolute Error } \\
\text { (Years) }\end{array}$ & $\begin{array}{l}\text { Median Absolute Error } \\
\text { (Years) }\end{array}$ \\
\hline $\begin{array}{l}\text { KBest 2000 de novo then } \\
\text { Boruta (35) }\end{array}$ & 0.928 & 3.39 & 2.92 \\
\hline Top 5 Most Frequent (5) & 0.911 & 4.02 & 3.72 \\
\hline
\end{tabular}

Table 4. Table showing the results of the two models created from the Horvath down syndrome blood

dataset (GSE52588, Horvath et al, 2015) using the same CpGs selected from the two feature selection

from the initial Hannum experiment. Number of $C p G$ sites/features in parentheses.

0.8

0.2

0.0

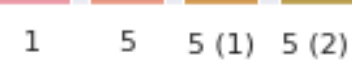

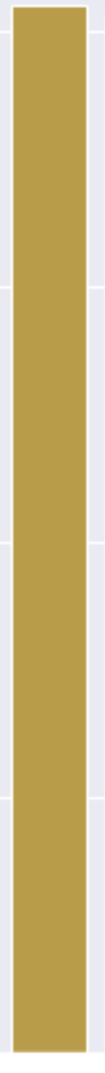

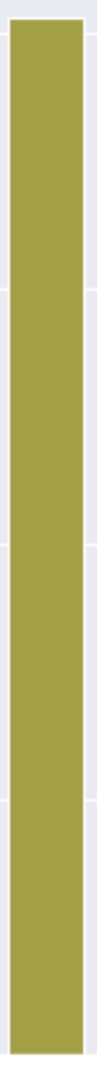

7

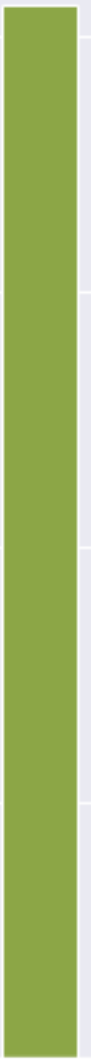

10
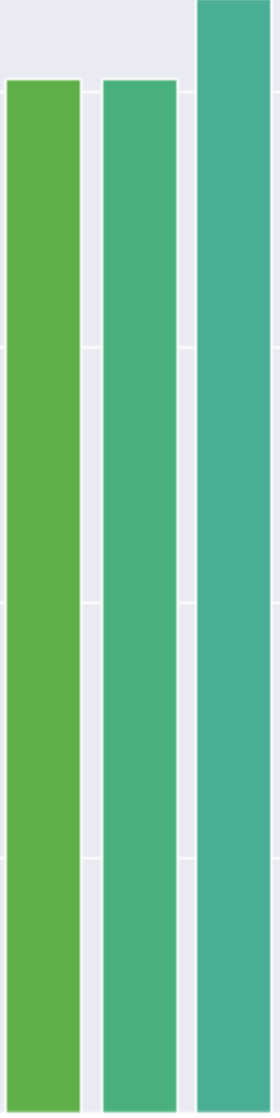

16
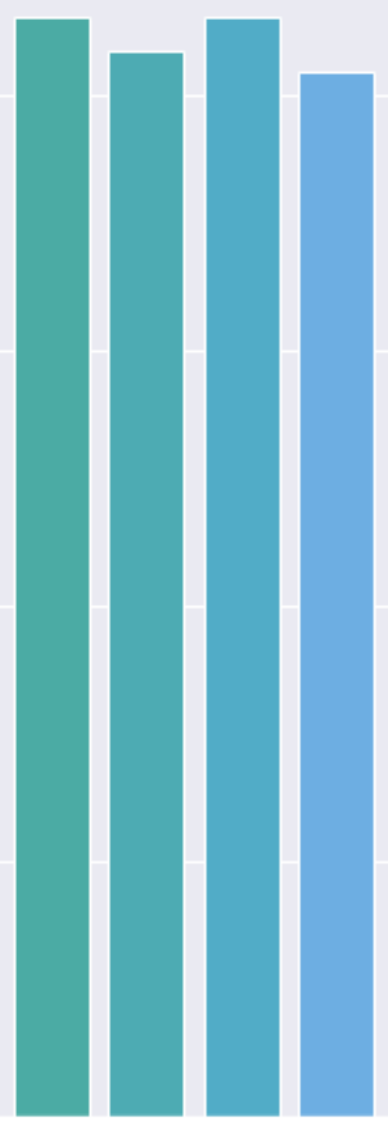

Feature Selection Methods 
bioRxiv preprint doi: https://doi.org/10.1101/2022.02 21.481326. this version posted February 22, 2022. The copyright holder for this preprint (which was not certified by peer review) is the author/funder, who has granted bioRxiv a license to display the preprint in perpetuity. It is made available under aCC-BY 4.0 International license.

Post Feature Selection Intersection of all methods (1)

SFM ExtraTrees de novo then Boruta (5)

SFM ExtraTrees de novo (5)

Top 5 Most Frequent (5)

SFM ElasticNet de novo then Boruta (7)

Top 10 Most Frequent (10)

SFM ElasticNet de novo (16)

\%-RFE de novo to 1500 then SFM (16)

KBest 2000 de novo then Boruta (35)

KBest 25 de novo (36)

\%-RFE de novo to 1500 then Boruta (52)

Boruta de novo (53)

\%-RFE de novo to 10000 then Genetic Algorithm (54)

Neural Network feature selection (65)

Genetic Algorithm de novo (85)

Intersection of all methods per CV fold then Boruta (102)

\%-RFE de novo to 100 (161)

ElasticNet de novo/No Feature Selection (276)

163 Figure 1. Figure showing the comparative methods with the number of features used in each model on

164 the $x$-axis and their average $R 2$ scores on the $y$-axis. $R 2$ scores are relatively similar across the board

165 despite the number of features needed for prediction varying widely. 

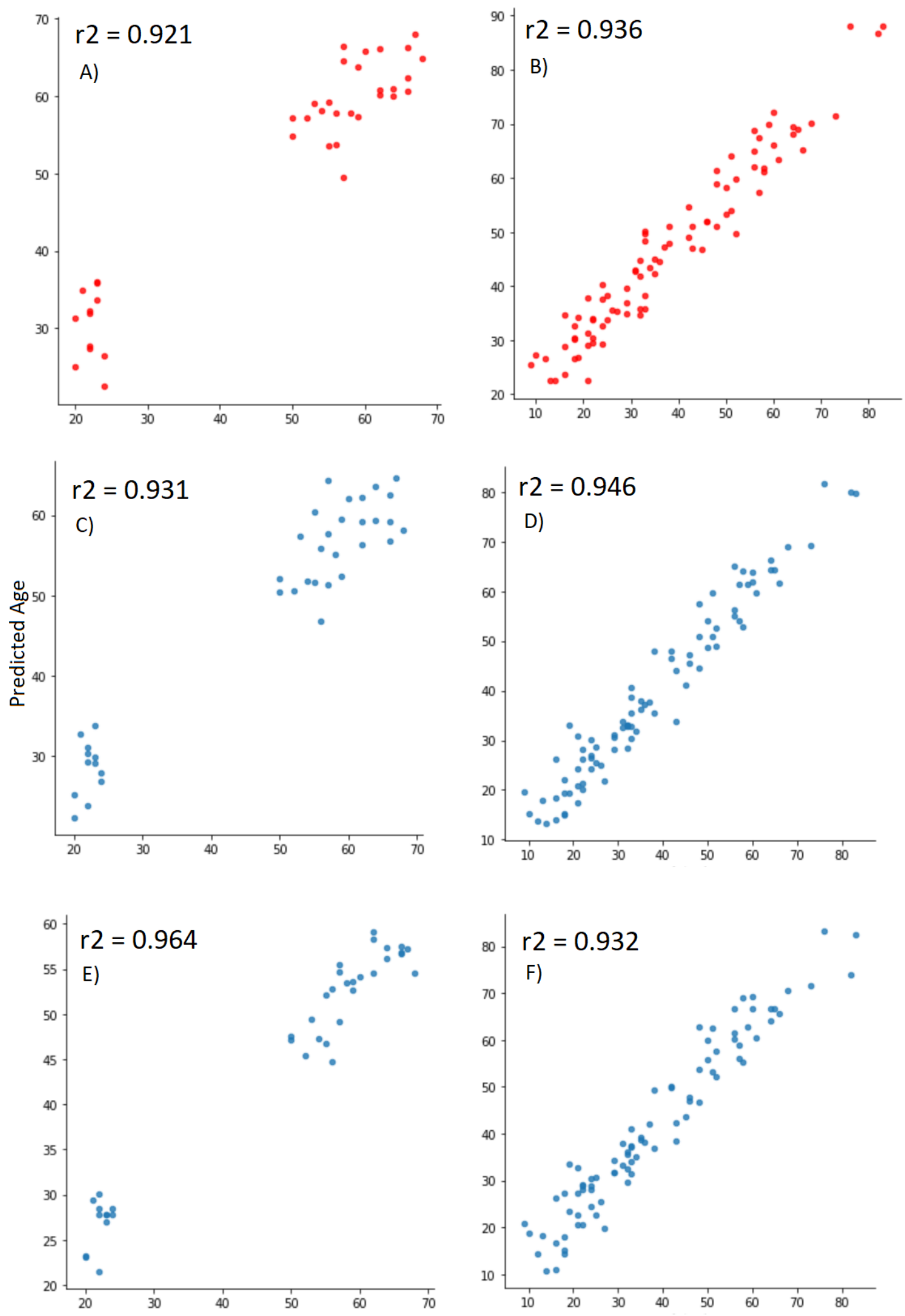
Figure 2. Figure showing the Predicted Ages vs Chronological Ages results of the two final models and Hannum's model on the two external validation datasets GSE85311 and GSE52588. (A-B) Hannum (CD) KBest 2000 de novo then Boruta (E-F) Top 5 Most Frequent.

\section{Discussion:}

Overall we show feature selection methods can select $\mathrm{CpG}$ sites that are highly predictive of age, allowing for less features needed to build an accurate epigenetic clock. Many different types of feature selection methods are able to attain a reasonably high correlation score of around $0.75-0.85$ whilst using a low number of $\mathrm{CpG}$ features. The rudimentary base code that outlines most of the feature selection ideas in this paper is publicly available and we hope that feature selection becomes a standard discrete step in future epigenetic clock studies. The corresponding genes of the most common CpG sites in these clocks are possible future targets for aging studies.

Two of our clocks, both trained on the original Hannum dataset, also performed well on two external datasets. The models, in fact, performed higher on validation datasets than the training dataset, and outperformed Hannum's original clock that uses 71 features. This validates both the feature selection methods' ability to reliably select good CpGs and the construction of our clocks. These clocks are thus able to be used by others reliably to serve as predictors of chronological age. We also applied these models to a dataset of a different sample type; buccal epithelial cells. Although the $r 2$ scores were only moderate for this dataset, the mean and median absolute errors were the lowest we observed. This suggests an interesting future potential for buccal/saliva methylation samples, as they are much more accessible and less expensive to obtain.

In addition to the validation of the clocks, we also tested whether the identified CpGs of two of these methods could be used to make accurate clocks using the Horvath down syndrome dataset (Horvath et al, 2015, GSE52588). These clocks still achieved high 0.91-0.92 r2 scores (Table 4). This suggests that 
these features and their ability to predict age are not dataset specific and can universally be used across other methylation datasets.

$$
\text { We identified five CpGs and their corresponding genes that were of particular interest, as they }
$$
were most commonly identified across all feature selection methods in our study (Table 3). Four of these CpG sites, and particularly ELOVL2, have been previously identified as strong predictors of age.

ELOVL2, C1orf132, FHL2 and CCDC102B are included in an online seven CpG site epigenetic clock from the University of Santiago de Compostela (Mathgene, 2021). Zbieć-Piekarska et al constructed a linear regression model using only ELOVL2's CpG site (cg16867657) to predict age (Zbieć-Piekarska et al., 2015) and obtained a high degree of accuracy in blood samples from humans. By manipulating the expression of ELOVL2 and observing age-related changes in the eyes of mice, Chen et al suggest that the gene is a molecular regulator of aging in the retina. Spólnicka and colleagues used ELOVL2 to accurately detect age differences from 3 disease groups (Spólnicka et al., 2018), and also highlight C1orf132 and FHL2 as key genes from which CpG sites are used for their epigenetic clock. CCDC102B also has links to aging and age-related degenerative diseases (Hosoda et al., 2018, Xia et al., 2018). Ito and colleagues developed a clock using only the CpG sites associated with CCDC102B and ELOVL2 (Ito et al., 2018) and are able to predict age with an $\mathrm{r} 2$ of 0.75 . Additionally, Fleckhaus et al.'s study develops a clock using 8 target regions, four of which are ELOVL2, FHL2, CCDC102B and C1orf132 (Fleckhaus etc al, 2020). These papers show that our feature selection methods are able to select the most age-predictive CpG sites, consistently with other studies.

OTUD7A is the fifth gene of interest that we identified with our methods, but is the least documented. One study has previously identified that high methylation rates of $\mathrm{CpG}$ sites associated with OTUD7A are correlated with age (Tharakan et al., 2020), and Yin et al. identified it as a potential regulator for neurodevelopmental disorders (Yin et al.,2018). The role of OTUD7A in aging, if any, is not well-known and should be explored further. 
We also applied a neural network method for feature selection in this study, but found it was not as powerful in terms of predictive accuracy as the other feature selection methods. However, this method did select many $\mathrm{CpG}$ features that were missed by our other conventional and novel methods. As neural network architecture becomes more advanced in its ability to read in larger datasets, the features it selects may eventually rival the accuracy of other methods. The features identified with neural networks may also give rise to new sets of $\mathrm{CpG}$ sites and genes worthy of study in aging.

The feature selection methods we introduce here overcome the common computational issues of stock selection methods and select a low-number of $\mathrm{CpG}$ sites whilst still yielding predictions of age that have high accuracy. These methods can be applied to a range of future studies developing epigenetic methods targeting specific $\mathrm{CpG}$ regions (Griffin et al. 2021) are another prime example. Lastly, these methods are not limited to the identification of $\mathrm{CpG}$ sites as features, and this pipeline could be used to identify features for biomarkers or clocks developed from a range of datasets (eg. metabolomics, microbiome, clinical data), and to predict a variety of age and health outcomes.

\section{Methods:}

237 Data

The datasets for this study are from the Gene Expression Omnibus database under the accession codes GSE40279, GSE85311, GSE52588 and GSE137688. The main dataset GSE40279 we test the feature selection methods on contains 656 samples (instances) of whole blood human methylation levels at 473,035 CpG Sites (features), matched to chronological ages. All analysis was done in Python 3. All related code outlining our methods is available on github (https://github.com/adamyli/CLK-MKR). 


\section{Cross-validation and overall approach}

The main workflow methodology is outlined in Figure 3. The original dataset was split into 10

246 folds for cross-validation (CV). For each set of training folds, every different feature selection method

was performed to select the optimal features within that training data. For every CV iteration, the

intersection of each feature selection method was also recorded and we performed Boruta on the

intersected features. For each of the feature selection methods, the resulting unique features from each

of the 10 iterations were collected into an aggregated list and entered into a final results dataframe. This

dataframe contains every unique feature selected by each selection method at each of the 10 iterations.

Post-feature selection processes were then performed. These include the intersection between the

results of all selection methods and ranking the top 5 and 10 most common features out of all the results.

The results from these two post-feature selection processes were also added to the Results Dataframe.

The original dataset was split into 10 folds again and for each column of the Results DataFrame, which

represents the unique selected features for every method, we reduced the dataset down to the selected

evaluated the model on the test data (20\%) using the $\mathrm{r} 2$ scoring metric. For each column the mean of the

$10 \mathrm{r} 2$ scores was the performance estimate of that feature selection method.

The best performing model was the clock from the SelectKBest method down to 2000 features

followed by Boruta resulting in 35 selected features. The second model of interest uses the top 5 most

frequently selected CpGs. These 2 models were validated using two external blood methylation datasets; 
bioRxiv preprint doi: https://doi org/10.1101/2022.02.21.481326; this version posted February 22,2022 . The copyright holder for this preprint (which was not certified by peer review) is the author/funder, who has granted bioRxiv a license to display the preprint in perpetuity. It is made available under aCC-BY 4.0 International license.

\section{START}
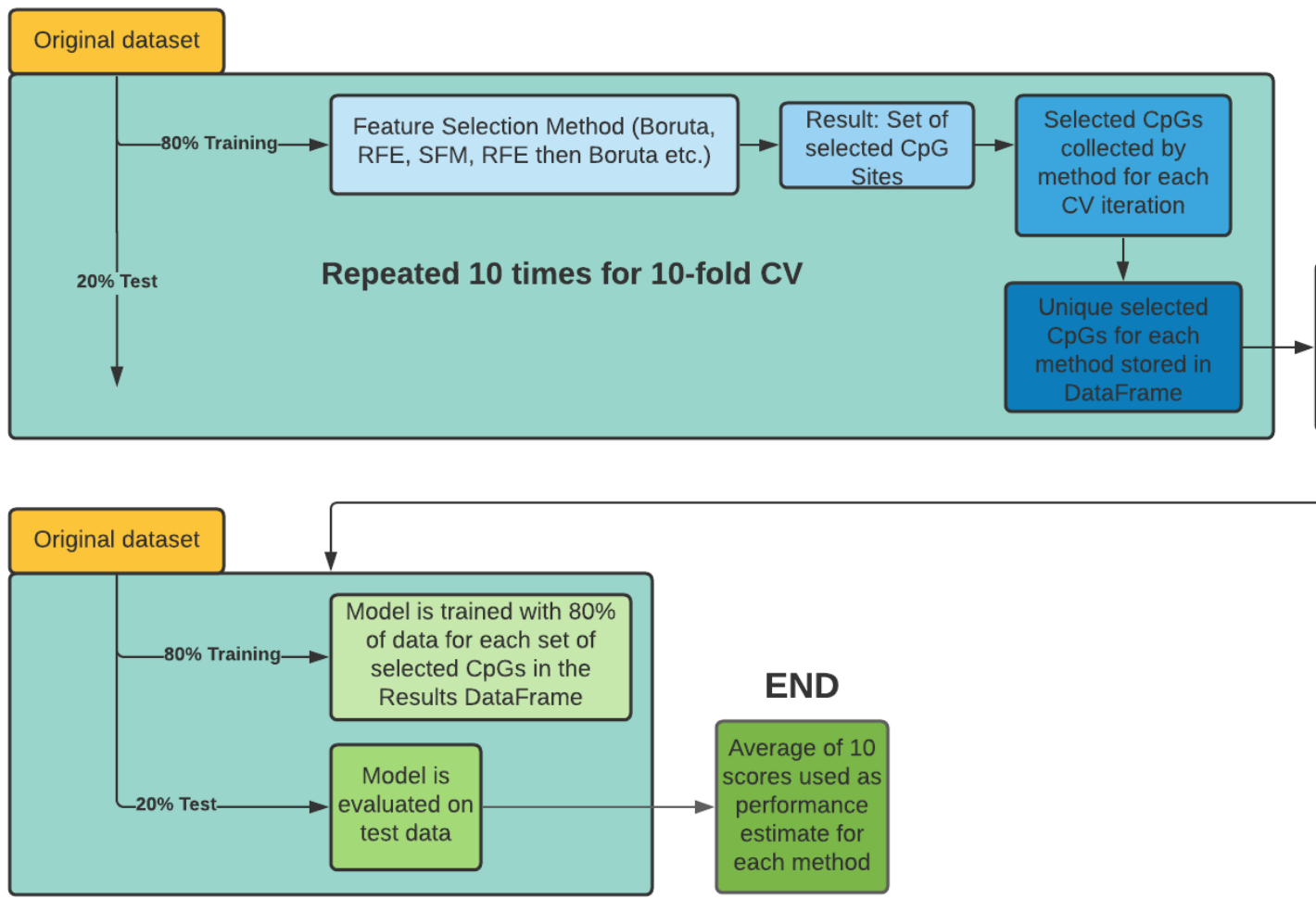

Repeated 10 times for 10 -fold CV

Figure 3. The workflow for feature selection and model evaluation. Feature selection was performed on training data for each iteration of 10-fold cross validation. The selected features of each iteration are aggregated into a list for each feature selection method type. The unique selected features for each method are collected into a dataframe where post-selection processes such as intersections are performed. We add the results to a dataframe. Each column of selected features in the results dataframe (each representing a different feature selection method) is tested using another training-testing split on the original data. This is done 10 times for 10-CV with the average of all scores being the performance estimate for that feature selection method. 


\section{SelectFromModel (SFM):}

SFM is a function within skLearn (Pedregos et al., 2011) that wraps around and trains a model on a dataset and allows the user to specify a threshold of feature importance. Depending on whether the model is a standard regression or random forest model, the feature importance is calculated from the coefficients or mean importance respectively. Features (CpG sites) with less feature importance than this threshold are discarded, leaving only the features with the highest coefficient or importance. This method is fast but simple. Thresholds of $0.01,0.05,0.1,0.5$ are tested. For this study, the models that the SFM wraps around are ElasticNet Regression and ExtraTrees forest.

The ExtraTrees Regression estimator is composed of a number of decision trees. A decision tree can be thought of as an intuitive flowchart where an answer to one decision between 2 or more choices leads to another. Decision trees decide how to split by prioritizing the split that creates the least uniform distribution of labels or values. This branching of nodes continues until it reaches a node that cannot decide which split to use because they result in equally uniform distribu-tions - meaning any more branches will not help the tree make any better decisions. In this sense ExtraTrees is similar to the more popular random forest with a few distinct differences. Random Forest samples the training data with replacement to train their decision trees whileExtraTrees uses the entire original dataset. However ExtraTrees randomly chooses the split instead of optimally finding a locally one which is what Random Forest does. ExtraTrees are therefore less exhaustive in their optimization and are faster than Random Forests. This is ideal for us as a Random Forest with 5-8 trees in it can take several hours to train on a dataset as large as ours. A random forest takes an advantage known as bagging by taking random instances of the dataset and training its model from solely those samples. For a regression problem like ours the average value of all trees are taken as the final prediction.

Recursive Feature Elimination (RFE) and the introduction of \%-RFE:

RFE is a function that trains a model on a dataset and removes the weakest feature based on the lowest feature importance from the dataset (Pedregos et al., 2011). This new dataset of N-1 features is 
trained again with a model and the process is repeated until only the user specified number of features is left. By removing 1 feature each time, RFE is a brute force algorithm that leaves only the best performing features at each iteration. However it does not take into account all features at the same time, and is unable to be aware of relationships between CpGs when it comes to predicting age e.g. some CpGs may become a strong predictor of ageing in the presence or absence of another.

Applying the stock RFE algorithm to our dataset of 473,035 features is computationally limiting due to the size of the dataset (Supplementary Table 1). Instead, we write an algorithm that removes a percentage-based number of features at each iteration allowing us to aggressively remove the majority of unnecessary features at the start but be more meticulous with our selection near the end. The percentage chosen is $1 \%$, i.e. removing 4730 features at 473,035 and 1 feature at 100 .

Boruta:

RFE is a 'minimal optimal' feature selection method, meaning it attempts to select the smallest set of features with the minimum error for an estimator and aims to optimize this ratio. Boruta differs as an 'all-relevant' feature selection method compatible with only tree-based regression methods, such as random forests (Kursa et al., 2010). Instead of trying to find the most compact set of features to predict with, it considers all features that could possibly contribute towards prediction overcoming the weakness of RFE's greedy nature. Boruta creates duplicates of the existing features with randomized values called 'shadow features'. The dataset comprising the original and the shadows, is trained on the tree estimator and the shadow features compete with their original forms. Features that consistently beat their shadow counterparts are selected as reputable predictors. In order to deal with the computational power needed to train a random forest with over 470,000 features, we use fewer trees and adjusted iteration counts in these models. 
SelectKBest is a feature selection method in sklearn similar to SFM that fits a dataset and selects features based on a scoring metric (Pedregos et al., 2011). For each feature it calculates the correlation value between the feature and target label and ranks them. This method is fast due to its shallow nature of only training once so is not useful when used alone. However, it is helpful to reduce the total number of features for usage of more greedy algorithms such as Boruta. In our methodology we select the top 25 features and the top 2000 features using SelectKBest. We perform Boruta on the top 2000 features.

Variance Threshold:

Variance threshold is a simple and exploratory method that removes all features whose column

of values do not reach the threshold of variance (Pedregos et al., 2011). Since some datasets naturally may not have a high degree of variance in their recorded data, this method is not consistent. However method.

RFE to reduce the number of features from 473,035 down to 100 . The NN first uses all 100 original and once where they all equal 0 to simulate the $\mathrm{CpG}$ being fully methylated and also absent. Both are done to account for the cases where the original methylation value is close to 0 or 1 . The mean of the two resulting scores are compared to the benchmark with the difference being recorded for each $\mathrm{CpG}$ site. 
a larger difference between the presence and absence of the $\mathrm{CpG}$ will insinuate that the $\mathrm{CpG}$ has a greater impact on age prediction. The top 50-75 are recorded as selected features.

Genetic Algorithm:

An algorithm based on the nature of Darwinism evolution where a population of 'creatures' are assigned a desired amount of features from the original dataset at random. These creatures are evaluated via predicting a validation set and assigned a score or 'fitness'. The lowest scoring creatures are culled next, simulating survival of the fittest. The remaining creatures are bred by creating a child creature that has features from their shared 'gene pool' and having a new number of them selected randomly. There is a chance for a certain number of these 'genes' to be mutated. Meaning some of the features will be randomly swapped for a different one from the original dataset. This helps introduce variation. This process is repeated for a specified number of generations or until a desired fitness is met.

The genetic algorithm is powerful as it allows the user many points of optimization, depending on the creativity of the user. For instance, the number of generations, number of features and creatures are all linked variables where a perfect balance can be found. When it comes to the breeding process it is possible to implement a 'polygamous' aspect where a highly successful creature is allowed to breed multiple times to ensure the most predictive features are passed on and tested further in other combinations. Mutation rate, number of genes allowed to mutate as well as number of children produced per breed (with possibility of scaling number of children produced with the fitness of the parent). It is also common for genetic algorithms to be run in parallel, predicting subsets of a label, e.g. an algorithm for young samples and one for old.

Novel methods combining multiple feature selection methods:

The introduction of \%-RFE and to a lesser extent SFM allows us to synthesize novel feature selection methods. \%-RFE allows for the removal of 'fluff' down to a more manageable number of features (usually a few thousand) and allows for more powerful methods to be used such as Boruta, 
Neural Networks and RFECV. These methods require more iterations and computational power so being able to distill down to the most important thousand features to choose from is ideal. The synthesized methods consist of \%-RFE first selecting features to an amount appropriate for the next method. SFM is also used as a preliminary selection method in this way. The final synthesized methods consist of modular code functions that allow us to alternate the order in which the selection methods are used as well as let us combine them together and use the output of onemethod as the input of another.

\section{Clock Models:}

The epigenetic clocks are built using ElasticNetRegression models. ElasticNet is chosen as it is the current standard for epigenetic clocks and outperforms Random Forests and SVMs with these data and feature selection methods.

This model is a variant of classical linear regression. This aims to solve for the coefficients of a linear equation that equals the 'best fit line'. The best fit line minimizes the sum of squares by having the least distance between the data points and the line. The equation for ordinary linear regression is as follows:

$$
\begin{gathered}
\operatorname{argmin}=\sum\left(y_{a}-y_{p}\right)^{2} \\
\operatorname{argmin}=\sum\left(y_{a}-\left(\beta_{1} x_{1}+\ldots \beta_{n} x_{n}\right)-b\right)^{2}
\end{gathered}
$$

Where $\mathrm{y} \_\mathrm{a}$ is the actual value of the target label and prediction $\mathrm{y} \_\mathrm{p}$ calculated by the summation of predictors ' $\mathrm{x}$ ' multiplied by a vector of coefficients $\beta \_n$ that is found from fitting the model $b . i s$ the $y$ intercept. argmin signifies a cost function where we seek to minimize the answer given input arguments. Regularization is a process in which different variants of bias and penalties are introduced to assist in finding the solution to this equation that allows for the best predictive accuracy. These penalties are controlled by a lambda value (alpha in sklearn) that controls how heavy (large) this penalty is. The L1 penalty is referred to as Lasso Regression, it adds a bias that is the absolute value of the coefficients. The L2 penalty is referred to as Ridge regression, this adds a bias that is the squared value of the coefficients. Unlike ridge regression, lasso regression can shrink the coefficients of unneeded parameters 
413 (features) to 0 (due to the penalty term not being squared), essentially eliminating them, leaving only useful features. Lasso can be quite aggressive however, taking only 1 feature out of several correlated ones or selecting too few. This is where ElasticNet comes in. The generic form of the ElasticNet equation is:

$$
\operatorname{argmin}=\sum\left(y_{a}-\beta x_{n}\right)^{2}+\lambda_{1} \sum|\beta|+\lambda_{2} \sum \beta^{2}
$$

Where L1 is the regularization penalty for the 'Lasso' part of the regression equation andL2 is the penalty for the 'Ridge' portion (Zou, Hastie. 2005). ElasticNet combines both Lassoand Ridge regressions, adding both terms to the equations. Each penalty gets an indepen-dent alpha / lambda that is tuned via cross-validation or other methods. This method allows the best of both worlds depending on the feature.

Acknowledgements: D.A.S. was supported by the Glenn Foundation for Medical Research; A.E.K was supported by a K99 award from the NIH (K99 AG070102); A.M. was supported by an F32 Ruth L.

Kirschstein National Research Service Award (NRSA) Individual Postdoctoral Fellowship from the NIH (F32 AG069363);

D.V. received financial support from the NIDDK Mouse Metabolic Phenotyping Centers

(RRID:SCR_008997, MMPC, www.mmpc.org) under the MICROMouse Funding Program, grants DK076169, and a NIH T32 grant T32AG023480.

Conflict of Interests: D.A.S. is a founder, equity owner, advisor to, director of, board member of, consultant to, investor in and/or inventor on patents licensed to Revere Biosensors, UpRNA,

GlaxoSmithKline, Wellomics, DaVinci Logic, InsideTracker (Segterra), Caudalie, Animal Biosciences, Longwood Fund, Catalio Capital Management, Frontier Acquisition Corporation, AFAR (American Federation for Aging Research), Life Extension Advocacy Foundation (LEAF), Cohbar, Galilei, EMD Millipore, Zymo Research, Immetas, Bayer Crop Science, EdenRoc Sciences (and affiliates Arc-Bio, Dovetail Genomics, Claret Bioscience, MetroBiotech, Astrea, Liberty Biosecurity and Delavie), Life 
Capital. D.A.S. is an inventor on a patent application filed by Mayo Clinic and Harvard Medical School

that has been licensed to Elysium Health. More information at https://sinclair.hms.harvard.edu/david-

sinclairs-affiliations.

\section{References:}

444 Bocklandt S, Lin W, Sehl ME, Sanchez FJ, Sinsheimer JS, Horvath S, et al. Epigenetic predictor of age.

445 PLoS One. 2011;6(6):e14821. doi:10.1371/journal.pone.0014821

447 Alghanim H, Antunes J, Silva D, Alho C, Balamurugan K, McCord B, 2017. Detection and evaluation of 448 DNA methylation markers found at SCGN and KLF14 loci to estimate human age. Forensic Science 449 International: Genetics, 31, pp.81-88.

Chen D, Chao D, Rocha L, Kolar M, Nguyen Huu V, Krawczyk M et al., 2020. The lipid elongation enzyme ELOVL2 is a molecular regulator of aging in the retina. Aging Cell, 19(2).

453

Darst B., Malecki K, Engelman C., 2018. Using recursive feature elimination in random forest to account for correlated variables in high dimensional data. BMC Genetics, 19(S1).

Pedregosa F, Varoquaux G, Gramfort A, Michel V, Thirion B, Grisel O, et al., Scikit-learn: Machine 
Graf GH, Crowe CL, Kothari M, Kwon D, Manly JJ, Turney IC, et al. (2021) 'Testing Black-White disparities in biological aging in older adults in the United States: analysis of DNA-methylation and

blood-chemistry methods', American journal of epidemiology. doi: 10.1093/aje/kwab281

Griffin PT, Kane AE, Trapp A, Li J, McNamara MS, Meer MV, et al. (2021) 'Ultra-cheap and scalable epigenetic age predictions with TIME-Seq', bioRxiv. doi: 10.1101/2021.10.25.465725.

Guyon I, Elisseeff A, An Introduction to Variable and Feature Selection:, Journal of Machine Learning

Hosoda Y, Yoshikawa M, Miyake M, Tabara Y, Shimada N, Zhao W, et al. 2018. CCDC102B confers methylation. Scientific Reports, 8(1). 
inflammation in the adipose tissue. Biochemical and Biophysical Research Communications, 497(3), pp.908-915.

Kursa MB, Rudnicki WR 2010. "Feature Selection with the Boruta Package.” Journal of Statistical

Levine ME, Lu AT, Quach A, Chen BH, Assimes TL, Bandinelli S et al. (2018). An epigenetic

biomarker of aging for lifespan and healthspan. Aging, 10(4), 573-591.

Lu A, Quach A, Wilson J, Reiner A, Aviv A, Raj K, et al. 2019. DNA methylation GrimAge strongly

predicts lifespan and healthspan. Aging, 11(2), pp.303-327.

Moore L, Le T, Fan G, (2012). DNA Methylation and Its Basic Function. Neuropsychopharmacology,

Mathgene.usc.es. 2021. Age prediction with DNA methylation: blood, 7 CpGs, EpiTYPER. [online]

Available from: http://mathgene.usc.es/cgi-bin/snps/age_tools/processmethylation-first.cgi.

Martens CR, Lubieniecki KL, McNamara MN, Bohr AD, McQueen MB, Seals DR, (2020) 'Epigenetic patterns with aging and exercise are associated with indicators of healthspan in humans' National Center 508 for Biotechnology Information. U.S. National Library of Medicine; [online] Available from: https://www.ncbi.nlm.nih.gov/geo/query/acc.cgi?acc=GSE85311 
McEwen L, O’Donnell K, McGill M, Edgar R, Jones M, MacIsaac J, et al. (2019). The PedBE clock

accurately estimates DNA methylation age in pediatric buccal cells. Proceedings Of The National

Academy Of Sciences, 117(38), 23329-23335. doi: 10.1073/pnas.1820843116

Meer MV, Podolskiy DI, Rashkovskiy A, Gladyshev VN (2018). A whole lifespan mouse multi-tissue

Meng H, Murrelle E, Li G, 2008. Identification of a small optimal subset of CpG sites as bio-markers

Park JL, Kim JH, Seo E, Bae DH, Kim SY, Lee HC, et al. Identification and evaluation of age-correlated

DNA methylation markers for forensic use. Forensic Sci Int Genet. 2016;23:64-70. doi:

10.1016/j.fsigen.2016.03.005.

525

Petkovich DA, Podolskiy DI, Lobanov AV, Lee SG, Miller RA, Gladyshev VN (2017). Using DNA 954-960.e6

Renner M, Wolf T, Meyer H, Hartmann W, Penzel R, Ulrich A, et al. 2013. Integrative DNA methylation and gene expression analysis in high-grade soft tissue sarcomas. Genome Biology, 14(12), p.r137. 
Spólnicka M, Pośpiech E, Pepłońska B, Zbieć-Piekarska R, Makowska Ż, Pięta A, et al. 2017. DNA

methylation in ELOVL2 and C1orf132 correctly predicted chronological age of individuals from three disease groups. International Journal of Legal Medicine, 132(1), pp.1-11.

Stubbs TM, Bonder MJ, Stark AK, Krueger F, von Meyenn F, Stegle O, et al. (2017). Multi-tissue DNA methylation age predictor in mouse. Genome Biology, 18(1), 1-14. Study. The Journals of Gerontology: Series A, 75(11), pp.2051-2055. 1-13. doi: 10.1038/s43587-021-00134-3. human skeletal muscle. Journal of Cachexia, Sarcopenia and Muscle, 11(4), pp.887-898. Methylation Signatures in Lung Adenocarcinoma. SSRN Electronic Journal,.

Weidner CI, Lin W, Koch CM., Eisele L, Beier F, Ziegler P, et al. (2014) ‘Aging of blood can be tracked by DNA methylation changes at just three CpG sites', Genome biology, 15(2), p. R24. doi: 10.1186/gb- 
Xia Y, Huang N, Chen Z, Li F, Fan G, Ma D, et al. 2018. CCDC102B functions in centrosome linker assembly and centrosome cohesion. Journal of Cell Science, 131(23), p.jcs222901.

Yin J, Chen W, Chao E, Soriano S, Wang L, Wang W, et al. 2018. Otud7a Knockout Mice Recapitulate

Many Neurological Features of 15q13.3 Microdeletion Syndrome. The American Journal of Human Genetics, 102(2), pp.296-308.

Zbieć-Piekarska R, Spólnicka M, Kupiec T, Makowska Ż, Spas A, Parys-Proszek A, et al. Examination of DNA methylation status of the ELOVL2 marker may be useful for human age prediction in forensic science. Forensic Science International: Genetics, 14, pp.161-167.

572

573 Zbieć-Piekarska R, Spólnicka M, Kupiec T, Parys-Proszek A, Makowska Ż, Pałeczka A, et al. 2015.

574 Development of a forensically useful age prediction method based on DNA methylation analysis. Forensic

575 Science International: Genetics, 17, pp.173-179.

Zou H, Hastie T, 2005. Regularization and variable selection via the elastic net. Journal of the Royal

Statistical Society: Series B (Statistical Methodology), 67(2), pp.301-320. 


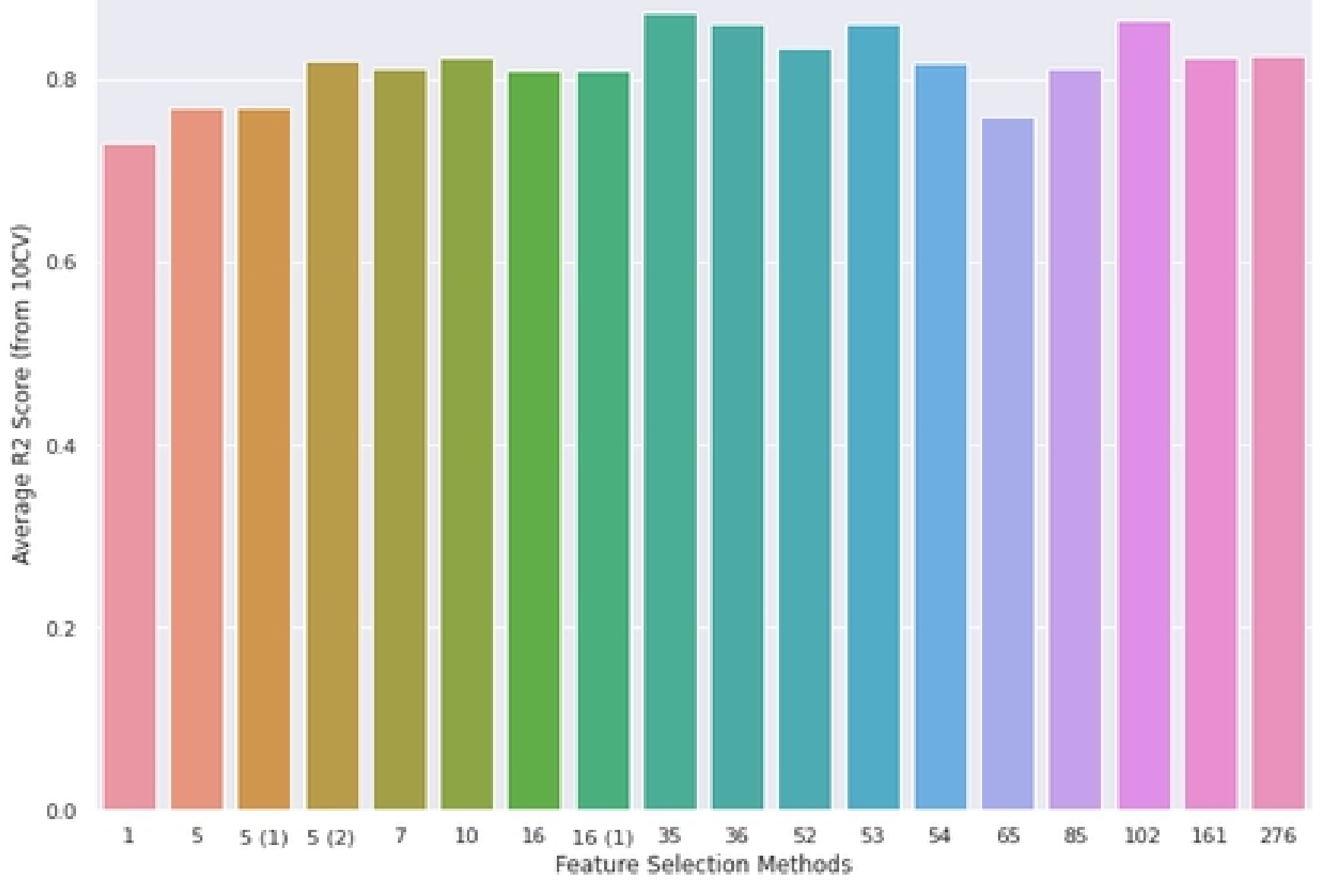

Post Feature Selection Intersection of all methods (1)

SFM ExtraTrees de novo then Boruta (5)

SFM ExtraTrees de novo (5)

Top 5 Most Frequent (5)

SFM ElasticNet de novo then Boruta (7)

Top 10 Most Frequent (10)

SFM ElasticNet de novo (16)

\%-RFE de novo to 1500 then SFM (16)

KBest 2000 de novo then Boruta (35)

KBest 25 de novo (36)

\%-RFE de novo to 1500 then Boruta (52)

Boruta de novo (53)

\%-RFE de novo to 10000 then Genetic Algorithm (54)

Neural Network feature selection (65)

Genetic Algorithm de novo (85)

Intersection of all methods per CV fold then Boruta (102)

\%-RFE de novo to 100 (161)

ElasticNet de novo/No Feature Selection (276)

\section{Figure 1}



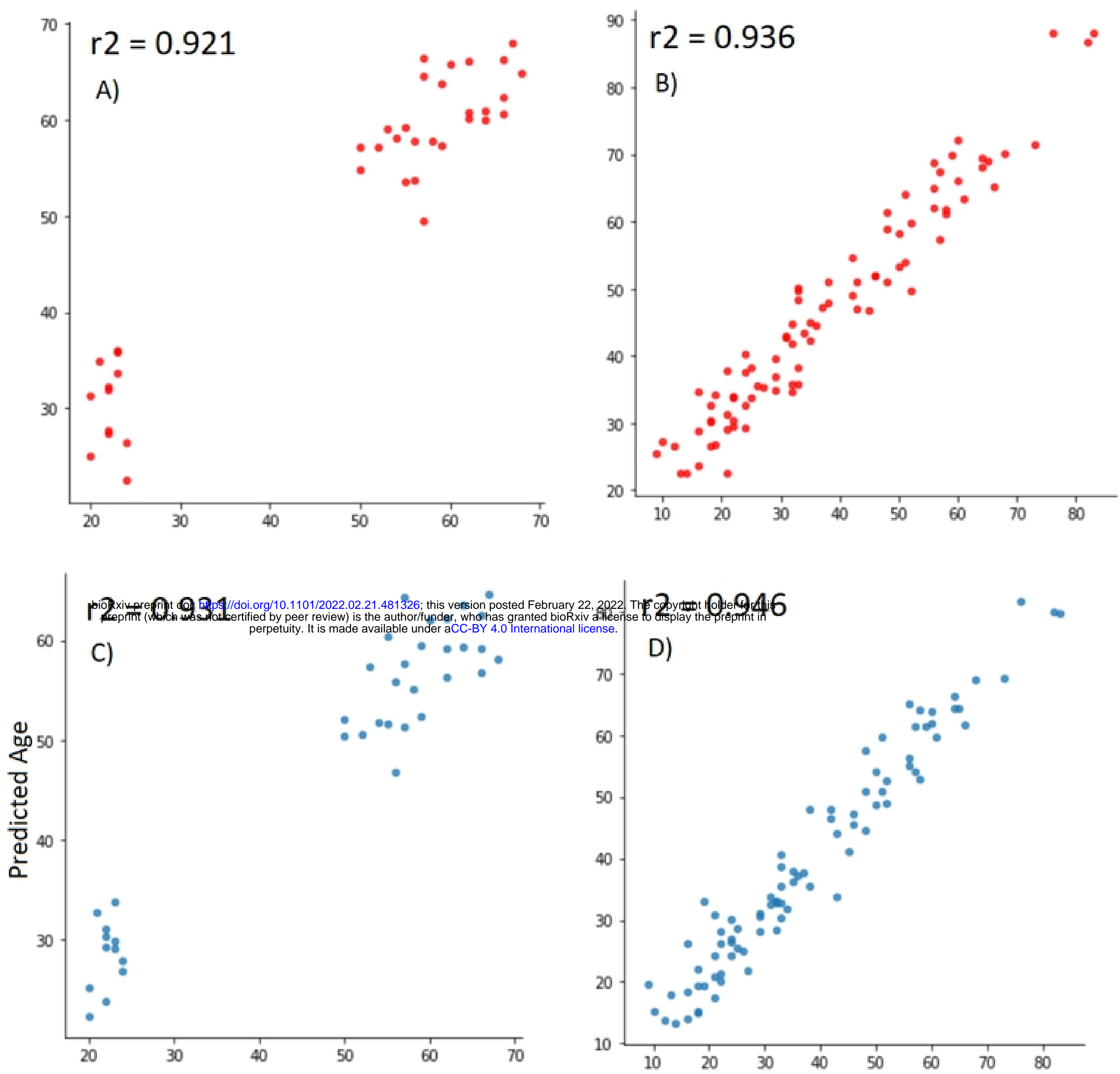

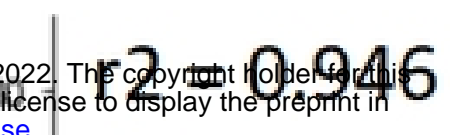
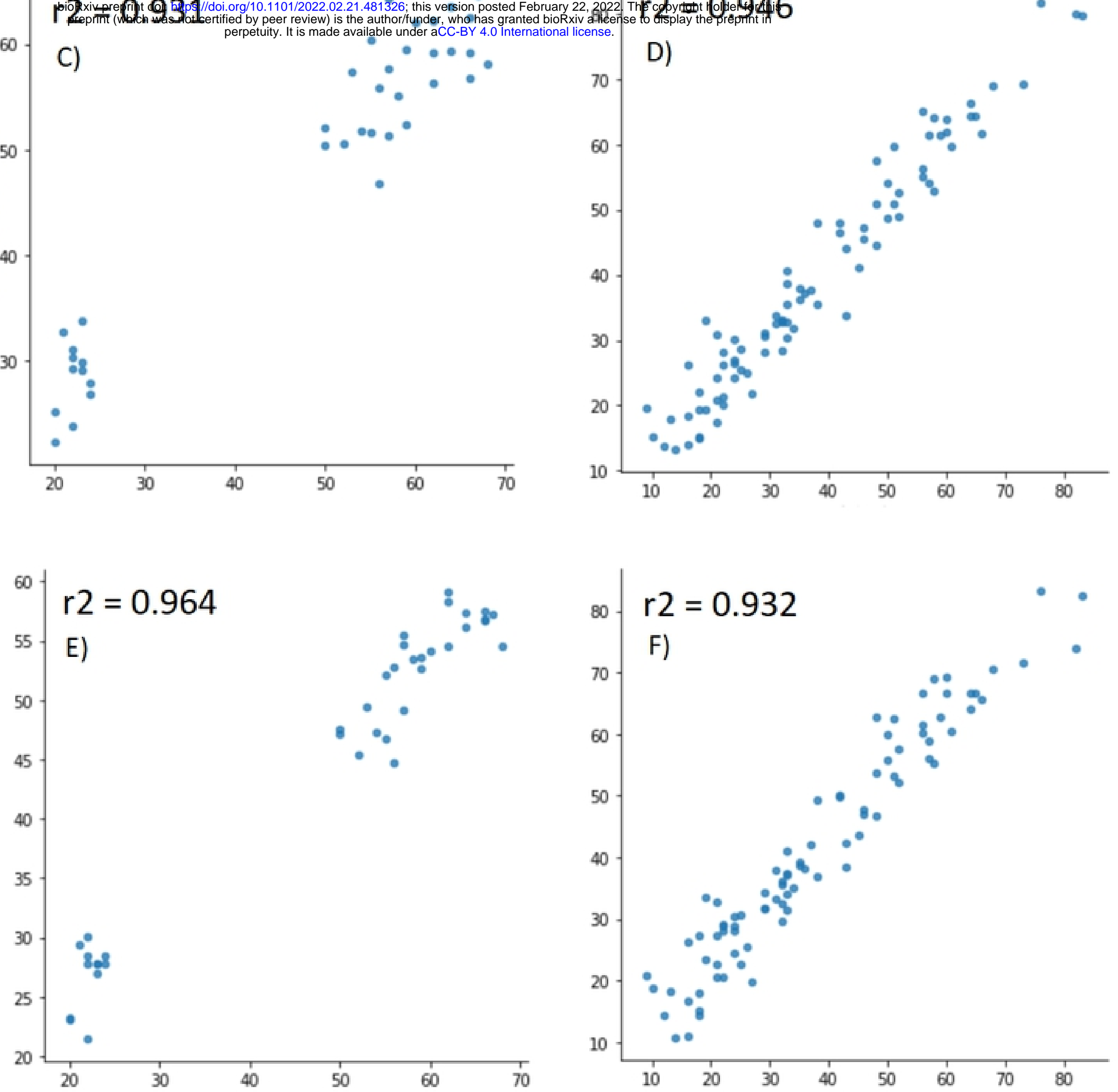

Chronological Age

\section{Cover Letter}




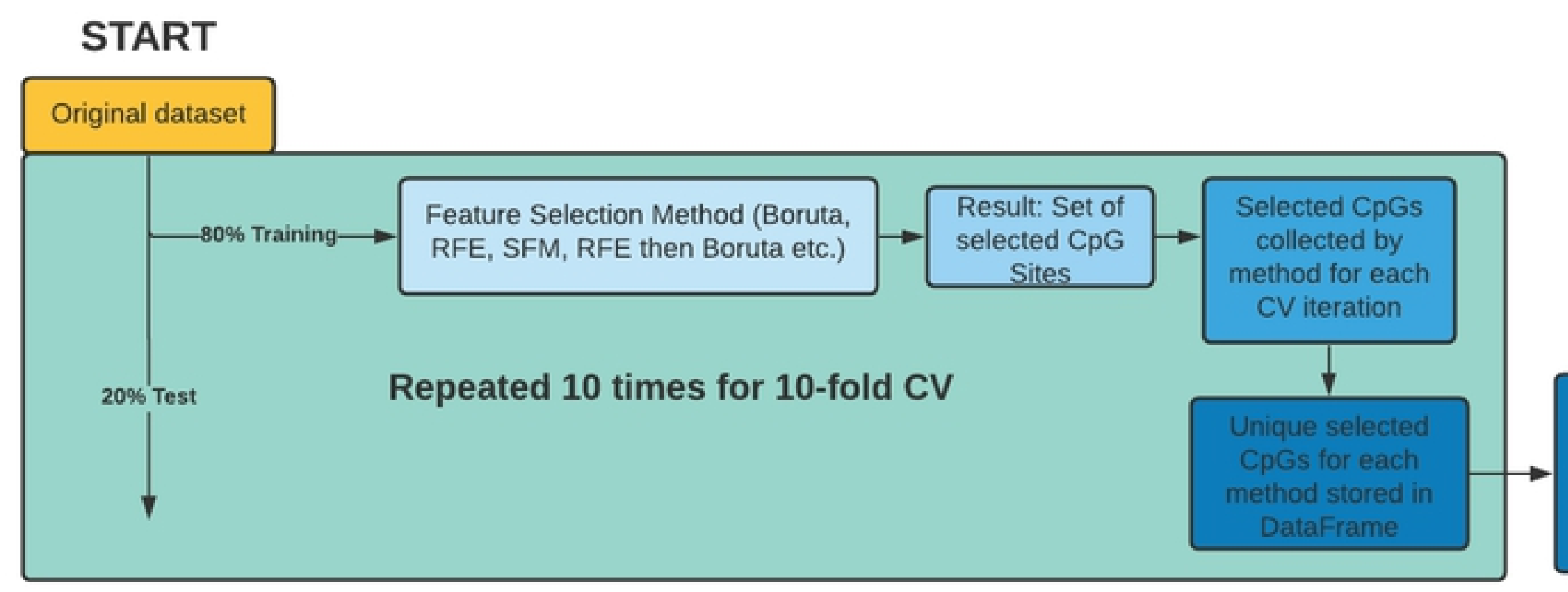

Post-selection processes such as frequency ranking and intersection are xecuted with results added to a Results DataFrame

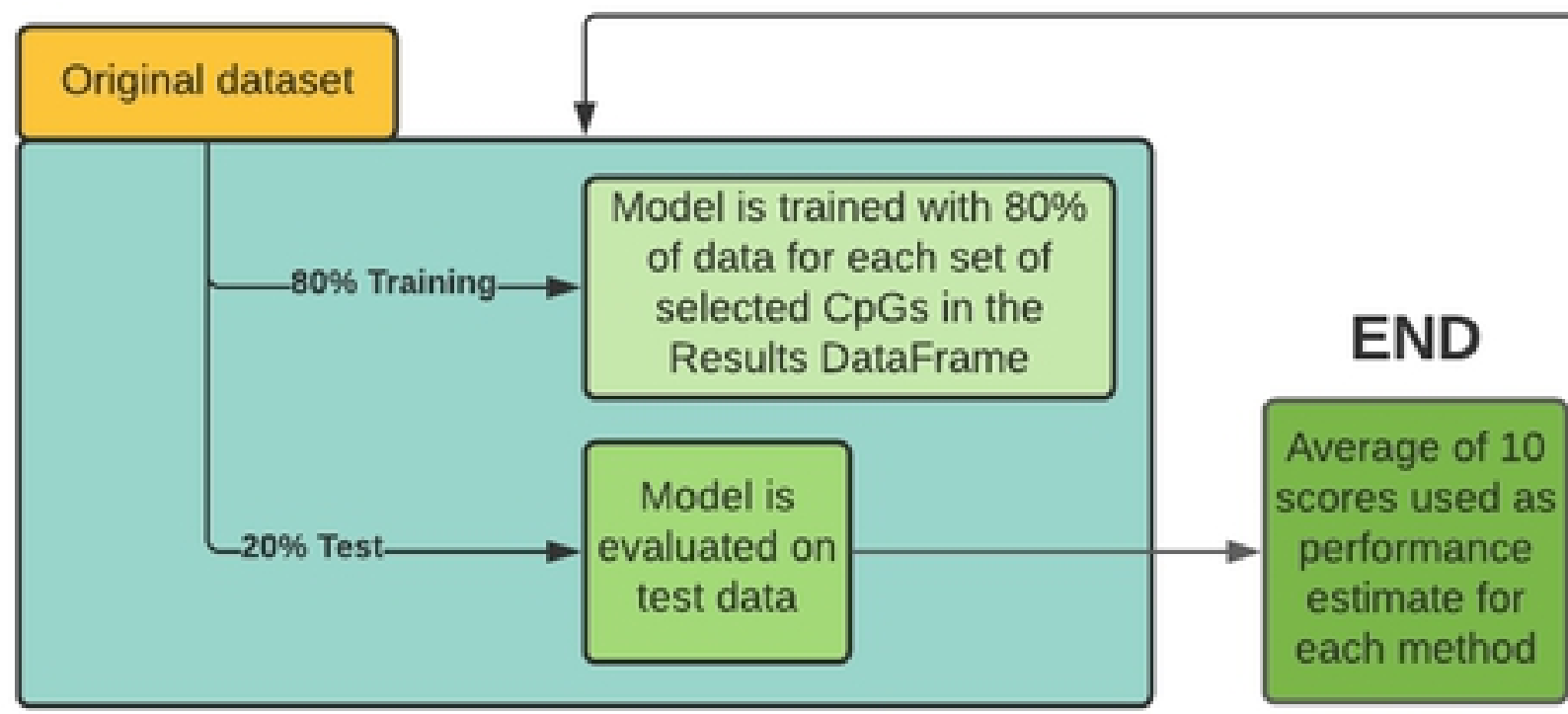

Repeated 10 times for 10 -fold CV

Figure 3 